
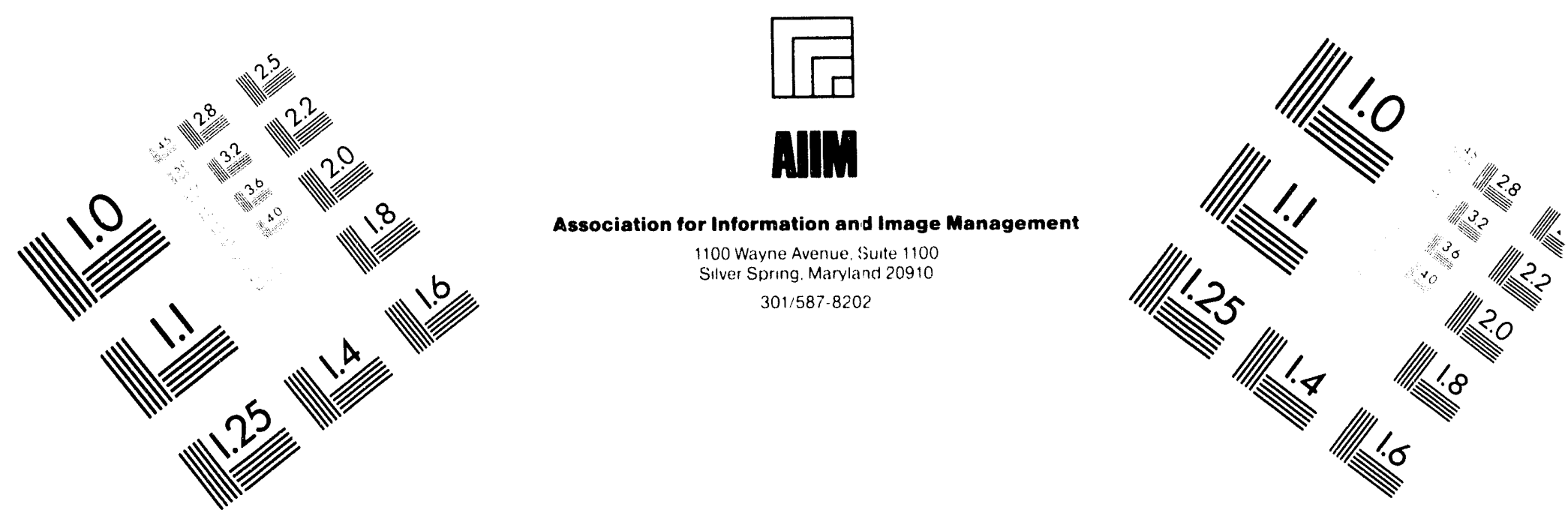

\title{
Centimeter
}

1

Inches
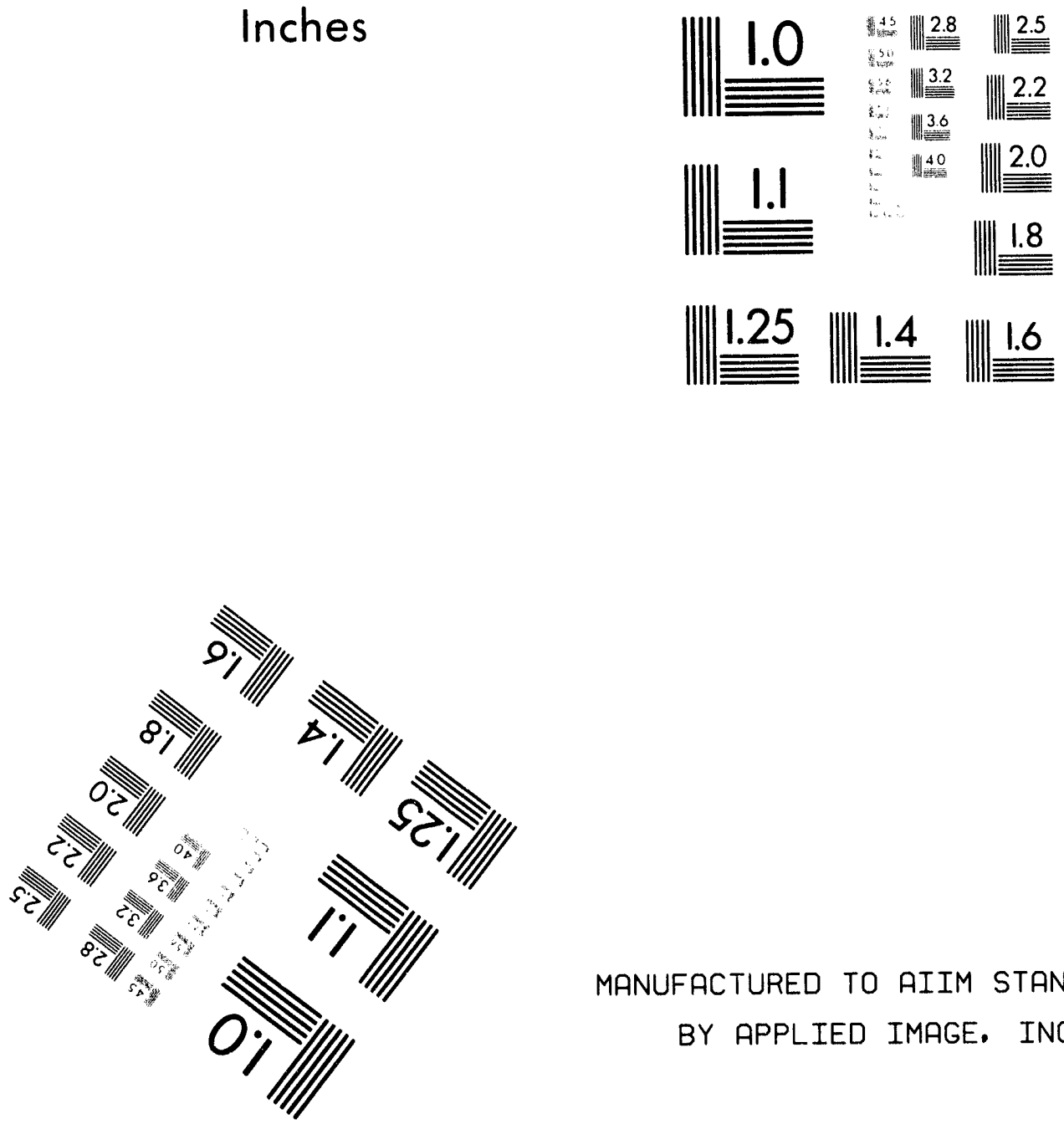

MANUFACTURED TO AIIM STANDARDS

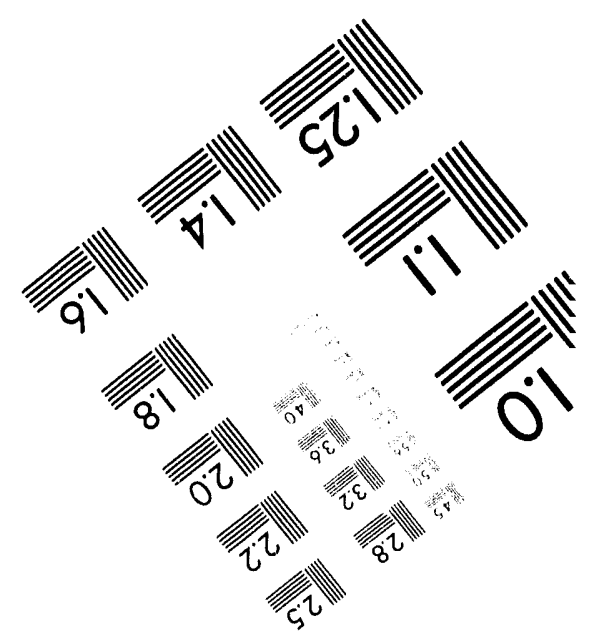



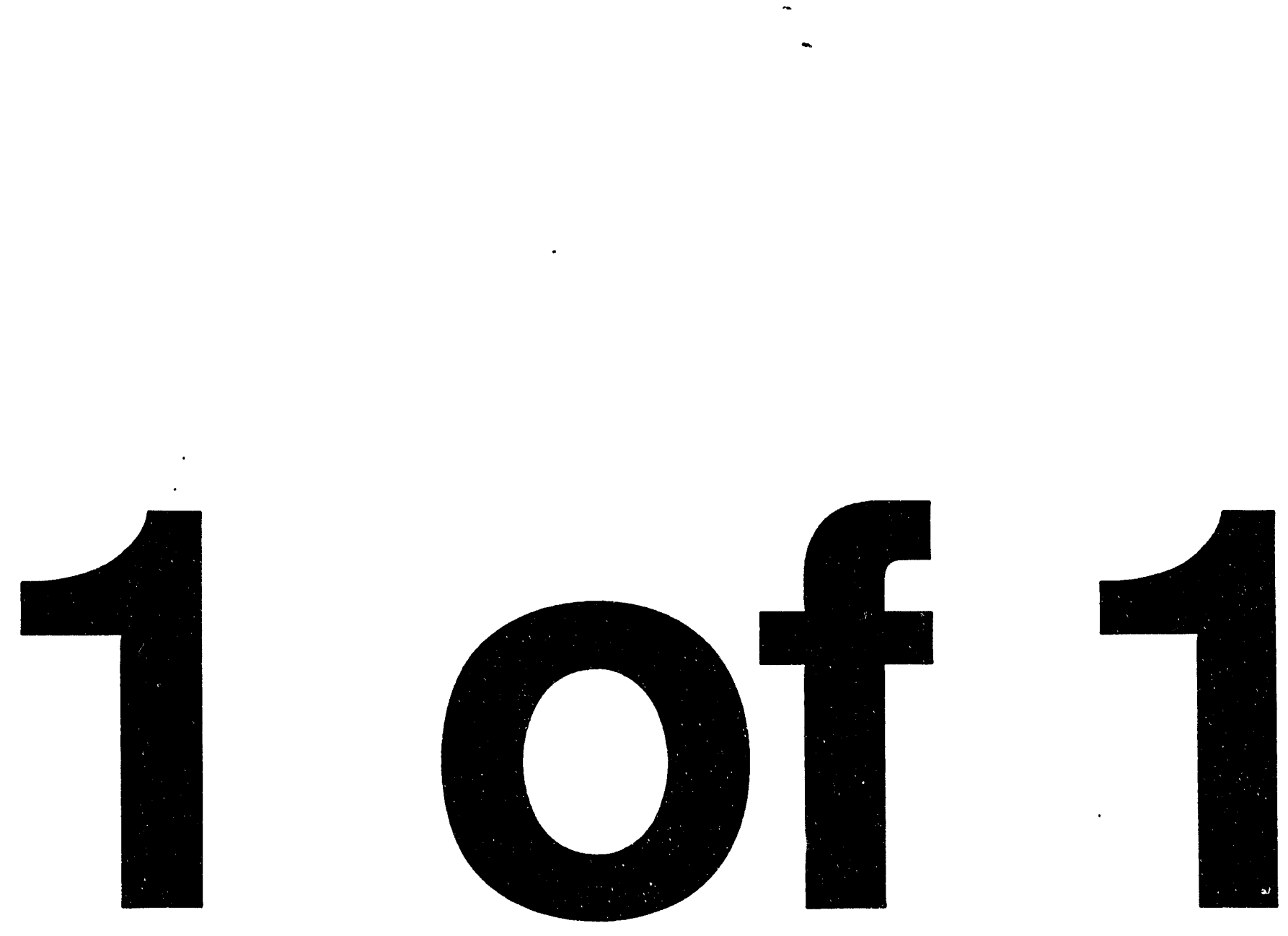


\title{
RESEARCH IN ACCELERATOR PHYSICS (THEORY)
}

Final Technical Report

\author{
May 15, 1993 - May 14, 1994
}

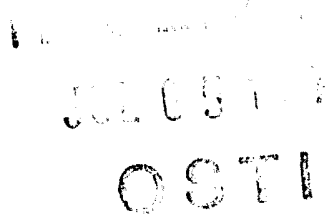

\section{Shoroku Ohnuma}

Principal Investigator

The University of Houston

Houston, Texas 77204-5506

June 8, 1994

PREPARED FOR THE U.S.DEPARTMENT OF ENERGY UNDER GRANT NUMBER DE-FG05-87ER40374 


\title{
I. INTRODUCTION
}

This is the final technical report of the grant DE-FG05-40374 covering the period from $5 / 15 / 93$ to $5 / 14 / 94$, the seventh year of the program which started in August 1987.

The most significant event during this period for the accelerator community is of course the termination of the SSC project. The collaboration with the SSC Laboratory through former members of the group, altogether six of them, came to the end with the decision to discontinue the effort at Waxahachie.

Our collaboration with Argonne National Laboratory, which started in June 1990, was also completed with the graduation of Yong-chul Chae in December 1993. The fifth Ph.D. from our group, he is now a member of the APS project at Argonne and working for the commissioning of the rings there.

With the termination of the SSC project, our new association with the LHC group at CERN (Walter Scandale and Frank Schmidt) will becorne more important for us than ever. Jicong Shi worked three months at CERN, June to August, in the area of nonlinear beam dynamics problems in their large hadron collider.

Our long association with the Texas Accelerator Center, which began in 1986, finally ended in August when Liming Xiu completedthe measurements on HESQ (Helical Electrostatic Quadrupole). It is our understanding that Charlie Meitzler and his group of the Sam Houston State University will continue the study of HESQ capability.

\section{DISCLAIMER}

\begin{abstract}
This report was prepared as an account of work sponsored by an agency of the United States Government. Neither the United States Government nor any agency thereof, nor any of their employees, makes any warranty, express or implied, or assumes any legal liability or responsibility for the accuracy, completeness, or usefulness of any information, apparatus, product, or process disclosed, or represents that its use would not infringe privately owned rights. Reference herein to any specific commercial product, process, or service by trade name, trademark, manufacturer, or otherwise does not necessarily constitute or imply its endorsement, recommendation, or favoring by the United States Government or any agency thereof. The views and opinions of authors expressed herein do not necessarily state or reflect those of the United States Government or any agency thereof.
\end{abstract}


Members of the group and their participations in the project during this period are:

S. Ohnuma (P.I.) Professor

J. Shi

G. Wang

L. Xiu

Y. Chae
SSC National Fellow

Sr. Research Assoc.

Res. Assist. Prof.

Research Associate

Research Scientist

$5 / 15 / 93-8 / 31 / 93$

Graduate Res. Assist. 5/15/93 - 12/31/93

$5 / 15 / 93-7 / 31 / 93$

$8 / 01 / 93-12 / 31 / 93$

$1 / 01 / 94-5 / 14 / 94$

$5 / 15 / 93-11 / 13 / 93$

$35 \%$ (cost sharing) during academic

year; 2 months in summer.

Reports made available during this period are:

DOE/ER/40374-

73. Progress Report, May 15, 1993 - October 31, 1993.

74. J. Shi, "Integrable Polynomial Factorization for Symplectic Systems", Phys. Rev. E (1994).

75. J. Shi and Y.T. Yan, "Explicitly Integrable Polynomial Hamiltonians and Evaluation of Lie Transformations", Phys. Rev. E48 (1993), 3943.

The doctoral dissertation by Yong-chul Chae completed during this period is "Longitudinal Coupling Impedance of a Hole in the Accelerator Beam Pipe", University of Houston, December 1993. 


\section{RESEARCH ACTIVITIES}

Status and main results from the following topics have been reported in Progress Report, $\mathrm{DOE} / \mathrm{ER} / 40374-73$, covering the first half of this project period:

1 Final Test of HESQ. (Xiu, Ohnuma) DOE/ER/40374-71.

2 Coupling Impedance of a Hole in Accelerator Beam Pipe. (Chae) Dissertation, University of Houston.

3 New Calculation of Diffusion Rate. (Shi). Report in preparation.

4 Integrable Polynomial Factorization for Symplectic Map Tracking. (Shi) DOE/ER/40374-75, Phys. Rev. E48, 3949 (1993)

5 Physics of Magnet Sorting in Superconducting Rings. (Wang, Shi, Ohnuma) DOE/ER/40373-78

During the second half of this project period, we have worked in the following areas:

1. Multi-Parameter sorting of Dipoles for Large Superconducting Rings

(Shi, Ohnuma, Wang), DOE/ER/40374-76

The presence of unavoidable multipole errors in superconducting magnets is known to be the main cause for limiting the dynamic aperture of large storage rings. Sorting of dipoles, in which dipoles are installed in the ring according to a certain sequence based on the measured multipole errors, is a way to reduce the adverse effects of random multipole errors without an increase in the cost. In order to reduce the effects of several multipole components simultaneously, we have developed a multi-parameter sorting scheme. In this sorting scheme, the multipole components are represented by a multi-dimensional vector and the sorting is based on the normal of the vector and the Euclidean distance between the vectors. Since the effects of different order of multipoles can not be directly compared by their multipole coefficients, the nonlinear field arising from each multipole is used as the sorting parameter. The nonlinear field associated with the $n$ th-order normal and skew multipoles at a phase-space location $x=y=x_{0}$ is characterized by $b_{n} x_{0}^{n}$ and $a_{n} x_{0}^{n}$ where $x_{0}$ will be used as a parameter to optimize the sorting. If the primary goal of sorting is to increase the dynamic aperture, the effectiveness of sorting is found to be peaked at $x_{0}$ 
near the dynamic aperture. Since the effects of these multipole components are taken into account simultaneously, this sorting scheme is very promising for the sorting of LHC dipoles, which has been a difficult problem due to the two-in-one design of dipoles.

To understand the effectiveness of our sorting scheme, we studied the dynamic aperture of a test lattice which is similar to the high energy booster (HEB) of SSC. To improve the statistical significance of our simulations, we have used a number of different samples of random multiple components, usually fifty, generated with different seed numbers in a random number generator routine. When the normal and skew sextupole components are important, the sorting are found to be very effective in that the dynamic aperture is more than doubled by the sorting. For the two-in-one design of dipoles, uncorrelated multipole components in two rings are sorted simultaneously and a similar improvement in the dynamic aperture has been observed. In order to understand the effect of high-order multipoles, we have also studied the system with all the high-order multipoles up to 12-pole included. Even though the sorting becomes less effective due to the large values of the high order multipoles, the multi-parameter sorting still has an advantage of the avoiding of the worst unsorted arrangement.

In order to examine the quality of a ring, it is desirable to have a set of figure-of-merits which can be evaluated without resorting to time-consuming tracking calculations. Search for the right figure-of-merits is however related to a long-standing unsolved question: how one can characterize the long-term stability of a nonlinear storage ring. So far, we have observed a definite correlation between the aperture and the harmonic components of resonance-driving integrals when the sextupole components are dominant. We have also studied the relation between the coefficients of one-turn Taylor map and the dynamic aperture. It has been found empirically that there exit a linear correlation between the aperture and the one-turn map coefficients (see Fig. 1). A complete understanding of this phenomenon is still under investigation.

It is known that a large nonlinearity in the ring can lead to a strong amplitude dependence of the tunes, which could result in crossings of dangerous resonances and a consequent reduction in the dynamic aperture. A weaker amplitude dependence of the tunes is therefore 
desirable for a stable operation of the accelerator. We have studied the effect of sorting on the amplitude dependence of the tunes by means of the normal forms. The preliminary results show that the sorting can reduce this dependence. Further studies on this are in progress.

\section{Integrable Polynomial Factorization for Symplectic Map Tracking}

(Shi), DOE/ER/40374-74

For large storage rings, extensive computer simulations are necessary to investigate the long-term stabilities. The conventional approach in which trajectories of particles are followed element by element through accelerator structure is rather time-consuming for these studies. A substantial computational as well as conceptual simplification is achieved by studying the stability with symplectic one-turn maps. It is, however, not clear how to construct an optimized symplectic one-turn map which has a similar long-term behavior as the original system and can be evaluated easily. During this project period, we have discovered that such a symplectic one-turn map can be constructed as a product of Lie transformations in the form of integrable polynomial factorization. With a system consisting of a single sextupole, the error involved in the integrable polynomial factorization map has been studied by comparing the results from the integrable polynomial factorization map with the results from exact map. It has been demonstrated that the error can be as small as one desires. desired. The long-term behavior of the integrable polynomial factorization map has also been tested by applying the map to various initial conditions. It has been found that the integrable polynomial factorization map has a similar long-term behavior as that of the original system even in regions where the system is quite nonlinear. Over the phase-space region of interest, the dynamics of a storage ring can be modeled by an integrable polynomial factorization map with a desired accuracy and the long-term tracking study can therefore be directly conducted with the map. 


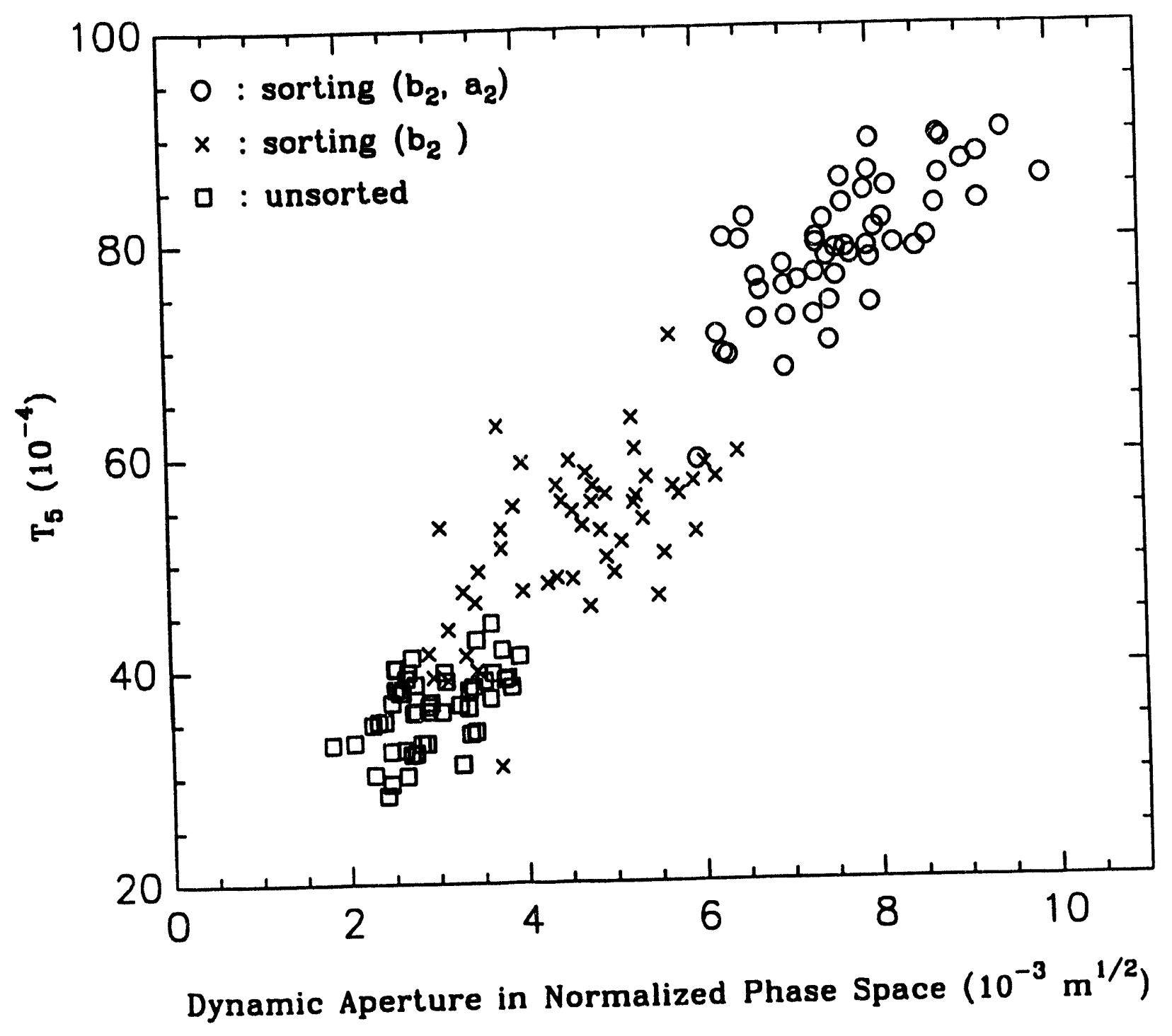

Fig. 1. Correlation between the dynamic aperture and the coefficients of one-turn Taylor map. $\sigma_{b_{2}}=\sigma_{a_{2}}=1.0 \mathrm{~m}^{-2}$ and other multipole components are absent. Squares represent fifty samples with random placement of dipoles; crosses represent the samples with only $b_{2}$ sorted; and circles represent the samples with both $b_{2}$ and $a_{2}$ sorted. $T_{N}$ is defined by

$$
T_{N}=(N-1) \sum_{n=2}^{N}\left\{\left[\sum_{i+j+k+l=n-1} C(i, j, k, l)\right] /\left[\sum_{i+j+k+l=n} C(i, j, k, l)\right]\right\}
$$

where $C(i, j, k, l)=\left|C_{x}(i, j, k, l)\right|+\left|C_{p_{\boldsymbol{e}}}(i, j, k, l)\right|+\left|C_{\mathbf{y}}(i, j, k, l)\right|+\left|C_{p_{y}}(i, j, k, l)\right|$ with $C_{x}(i, j, k, l)$, $C_{p_{z}}(i, j, k, l), C_{y}(i, j, k, l)$, and $C_{p_{y}}(i, j, k, l)$ denoting the coefficient of the term $x^{i} p_{x}^{j} y^{k} p_{y}^{l}$ in the map for $x, p_{x}, y$, and $p_{y}$, respectively. 


\section{OTHER ACTIVITIES}

S. Ohnuma visited KEK in Japan, Tsinghua University and Institute of High Energy Physics in Beijing, China, in May1993. The trip report for this has already been submitted to DOE.

Ohnuma spent one month in summer of 1993 at the SSC Laboratory working on the chromaticity correction in HEB with normal sextupole changing in a complex manner during the magnet ramping. An informal report on this work has been issued from the SSC Laboratcry.

He also participated in the workshop at Fermilab on Tevatron luminosity improvement, giving a talk on a new way to estimate the beam diffusion rate by J. Shi.

J. Shi spent three months, June to August 1993, at CERN working with the LHC group there. The visit was supported by the Texas National Research Laboratory Commission with a partial support from CERN. 




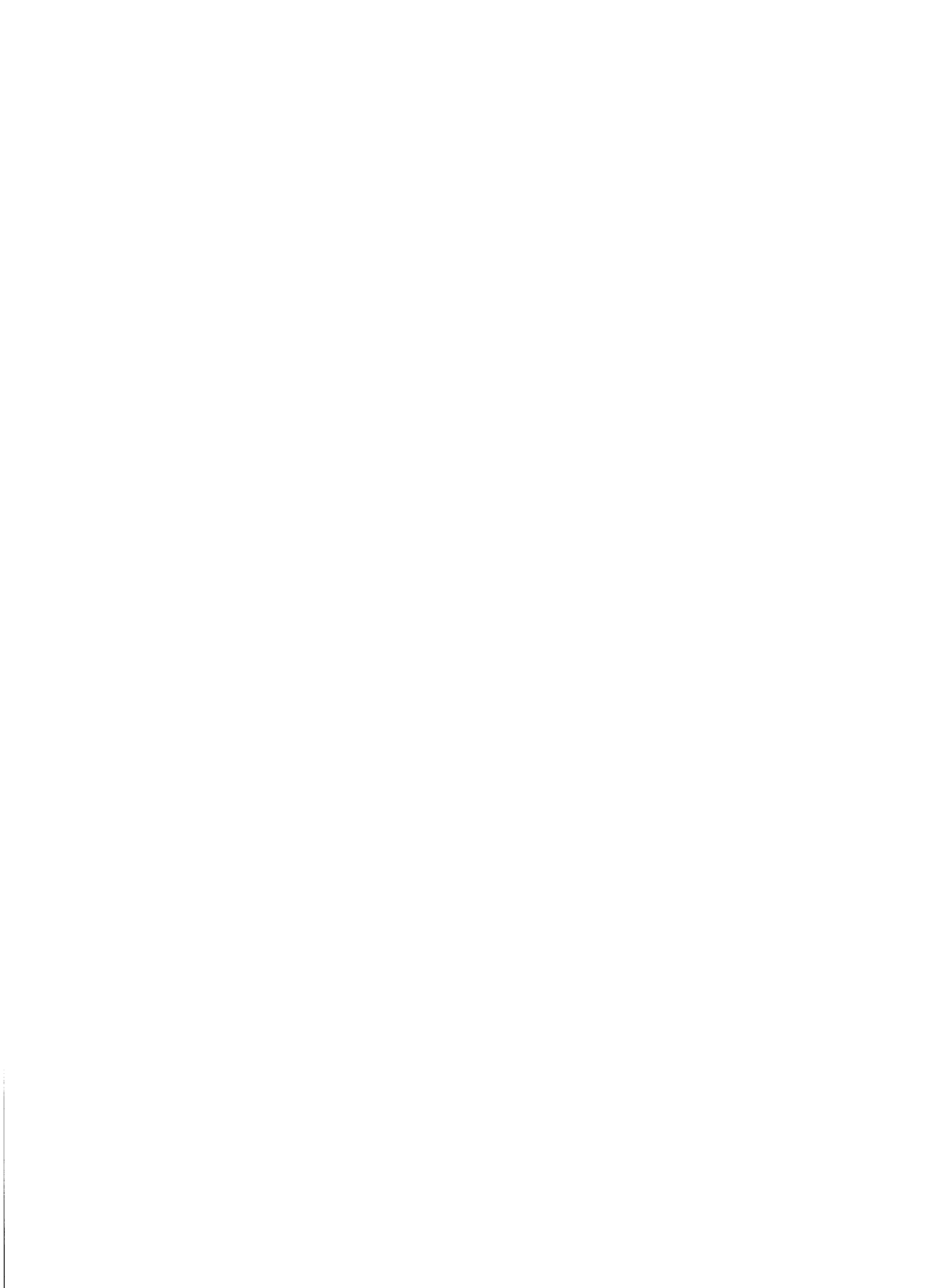

